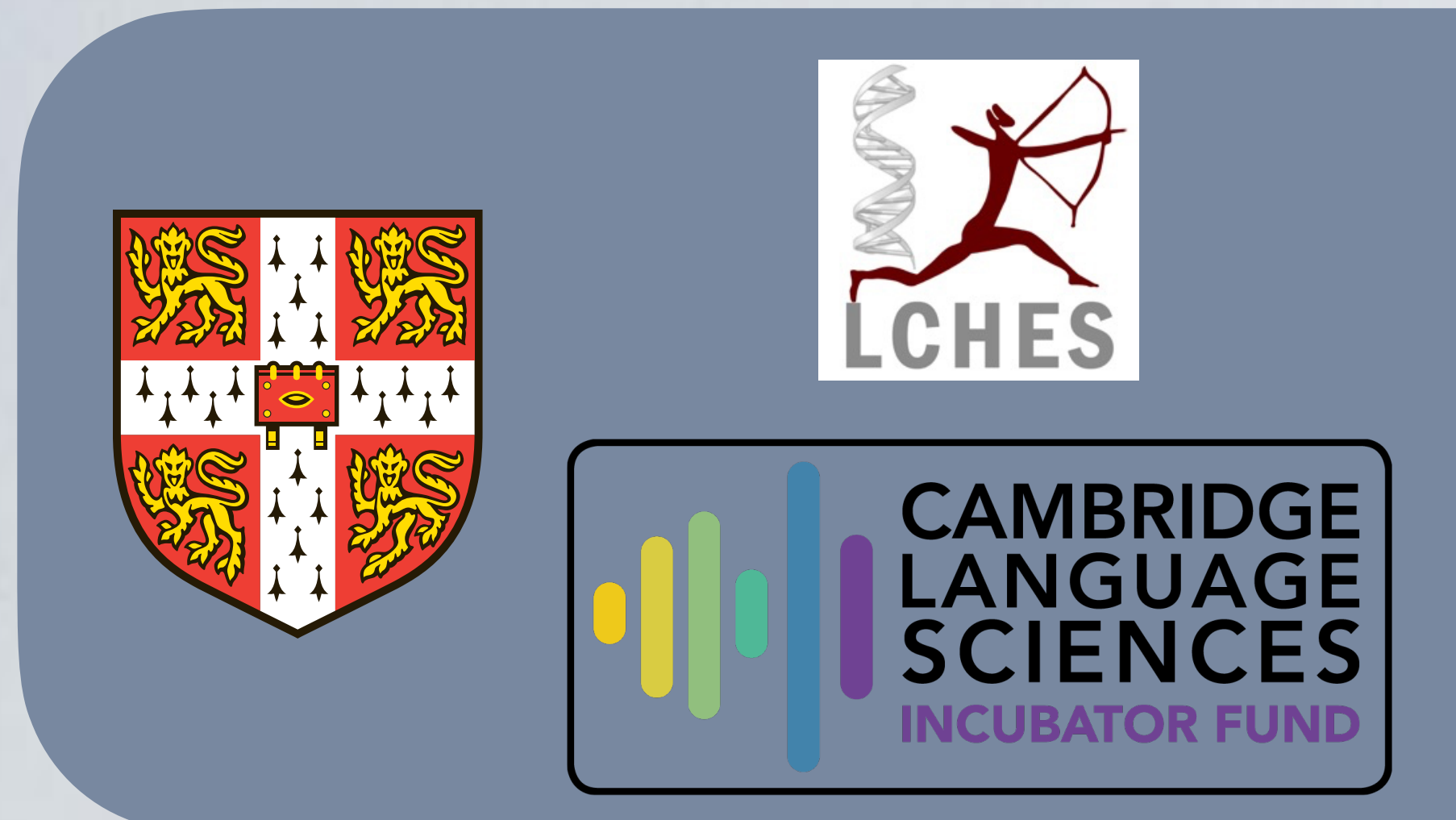

\title{
Accents as honest signals of in-group membership
}

\section{Jonathan R. Goodman'1, Enrico Crema1', Francis Nolan², Emma Cohen³, Robert Foley}

1. Leverhulme Centre for Human Evolutionary Studies $\quad$ 2. Phonetics Lab, MMLL $\quad$ 3. Social Body Lab

\section{What are accents for?}

Previous research (for example, see Dunbar, 2017; Nettle and Dunbar, 1997) suggests that language evolved partly as a mechanism for establishing closer ties within groups of increasing size in our evolutionary history.

Accents, along with other cultural features including shared place of origin, helped to increase the number of people with whom an individual could signal cooperative tendencies (Cohen, 2012).

Yet as groups became larger and underwent continued fission and fusion, signals of group membership may have become more important to reduce the risk of infiltration (Foley, 2004).

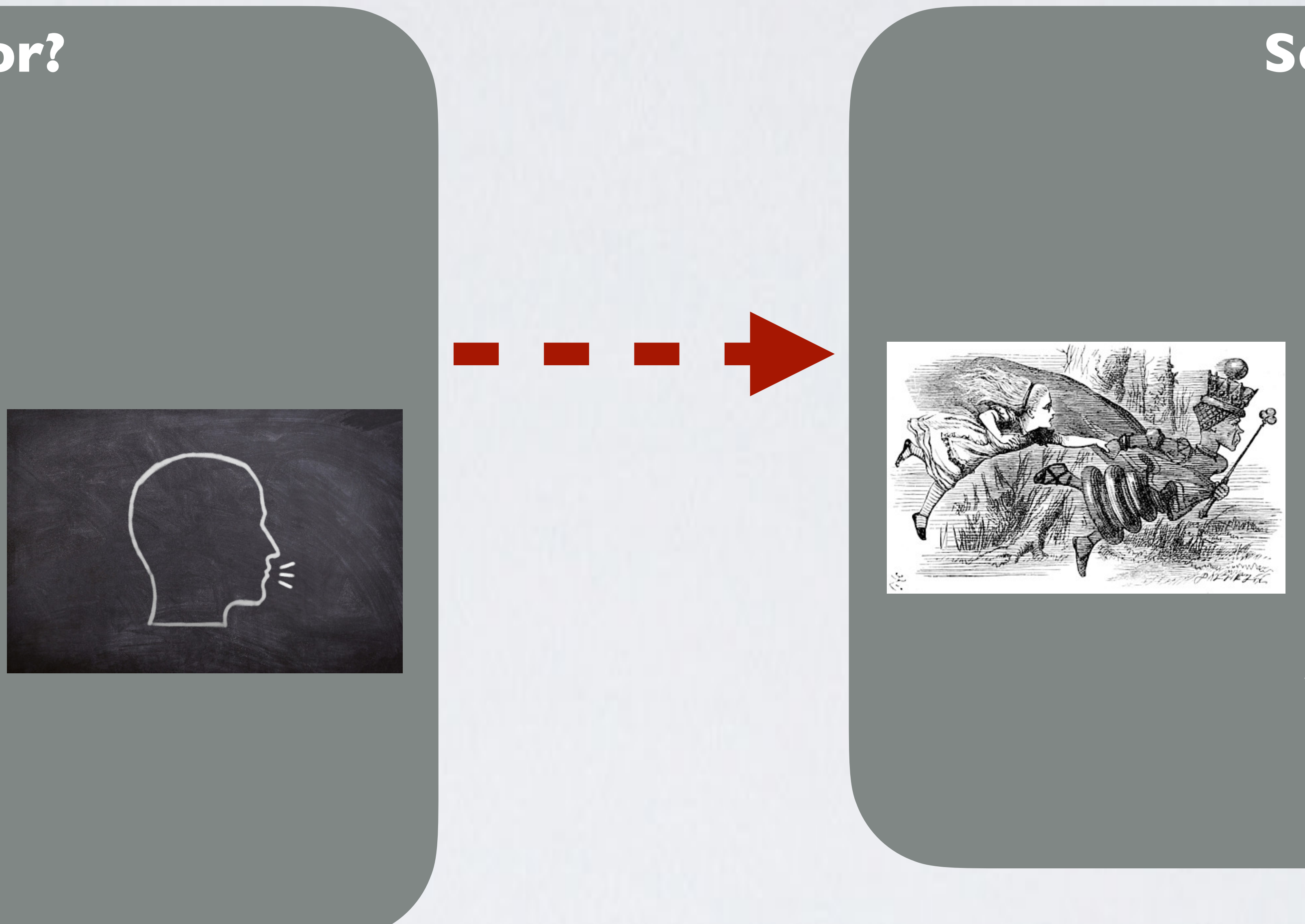

So why not fake it?

We would expect, as the risk of imposters grew along with group size, for signals of group membership to become more complex, and for true group members to become adept at recognising false signals (McElreath et al., 2003).

This suggests that, even before spoken language evolved, signallers and receivers were locked in a competitive relationship (Dawkins and Krebs,

1978). Because of the range of possible signals that the human vocal tract can produce, even without spoken language, we would expect a coevolutionary arms race to select for both strong mimicry and mimicry detection.

\section{Sentences and participants}

In this first phase, we are asking participants to:

A) Record themselves reading out a set of phonetically designed sentences;

B) Attempt to mimic recordings created by participants who speak naturally in one of the other accents in which we are interested; and

C) Listen to a set of 12 recordings of participants speaking in the listener's native accent, 6 of which are mimics and 6 of which are genuine accent-speakers, and to attempt to determine which are mimics and genuine-speakers.

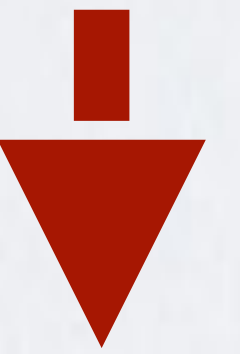

Analyses and results

We evaluated the overall probability of correct responses using a binomial test. We then created a hierarchical model, using participant as a random-level effect, and used Bayesian Markov Chain Monte Carlo sampling to determine the probability of correct responses by listener accent.

Thus far, 50 participants have consented to participate and completed all three tasks. The median participant age is 41 years (range, 18-83). By accent, these included 5 speakers of the Belfast accent, 6 speakers of the Bristol accent, 9 speakers of the Dublin accent, I I speakers of the Essex accent, 7 speakers of the Northeast England accent, and 12 speakers of the RP accent.

Overall, listeners had a $66.7 \%$ probability of correctly identifying a mimic and genuine speaker $(95 \%$ Cl: $0.658-0.675)$.

\section{Hypotheses and study design}

In the first part of this study, we are exploring how well people who speak naturally in 7 specific regions of the British Isles detect mimicry of their own accent. The accents we are evaluating include:
Bristol
Belfast
Dublin
Essex
Glasgow
Northeast England
Received Pronunciation (RP)

We are hypothesizing that speakers across regions will be better than chance at detecting accent mimicry.

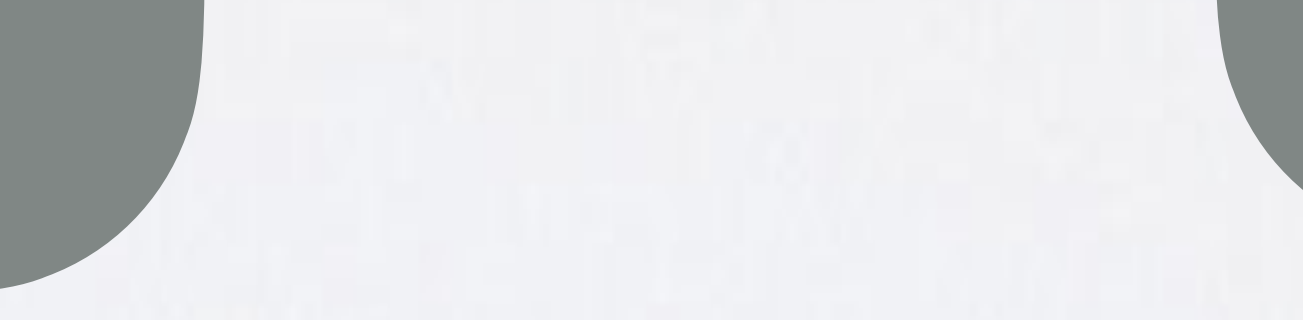

(
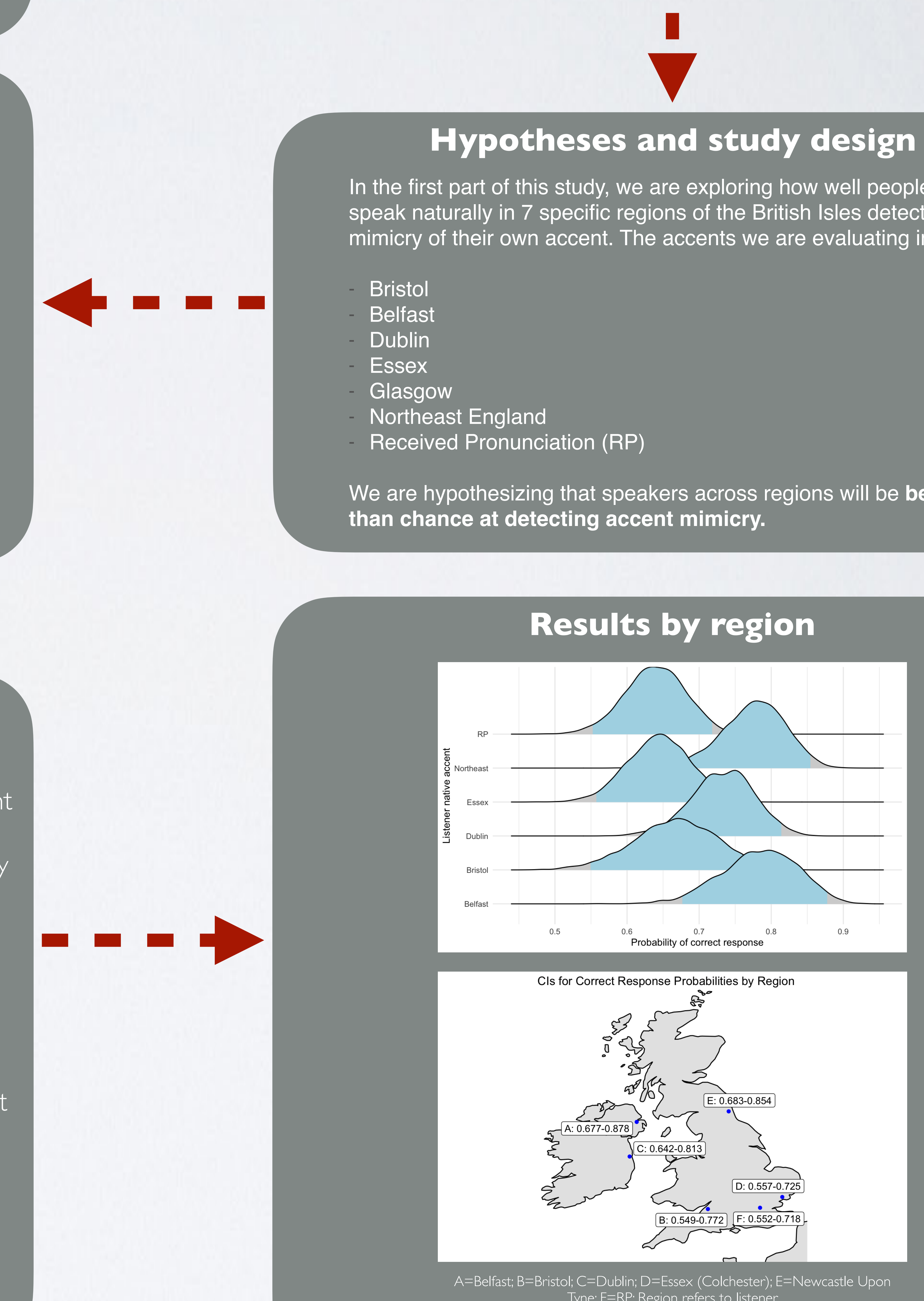

\section{Conclusion and future directions}

Overall, these results support our hypothesis, and suggest that individuals are better than chance at detecting accent-mimicry of their own native accents, supporting the evolutionary account of why we speak in accents.

The next phase of our study will evaluate how well individuals detect mimicry of accents that listeners do not speak natively. 